\title{
Medial Collateral Ligament Knee Sprains in College Football
}

\section{Brace Wear Preferences and Injury Risk}

\author{
John P. Albright, ${ }^{\star} \dagger$ MD, John W. Powell, ${ }^{\star}$ PhD, ATC, Walter Smith, $\ddagger$ MED, ATC, \\ Al Martindale,§ ATC,R, Edward Crowley, ${ }^{*}$ LPT, ATC, Jeff Monroe,\| MS, ATC, \\ Russ Miller, ${ }^{a}$ PT, ATC, John Connolly, ${ }^{b}$ PT, ATC, Billy A. Hill, ${ }^{c}$ ATC, \\ Dennis Miller, ${ }^{d}$ MS, ATC, PT, Dennis Helwig, ${ }^{e}$ ATC, and Jim Marshall, ${ }^{f}$ ATC, RPT
}

\begin{abstract}
From ‡Indiana University, §University of Illinois, *University of lowa, ||Michigan State University, ${ }^{a}$ University of Michigan, ${ }^{\prime}$ University of Minnesota, ${ }^{b}$ Northwestern University, ${ }^{c}$ Ohio State University, ${ }^{d}$ Purdue University, and ${ }^{\mathrm{e}}$ University of Wisconsin
\end{abstract}

\begin{abstract}
In this prospective, multiinstitutional analysis of medial collateral ligament sprains in college football players, we categorized 987 previously uninjured study subjects according to frequency of wearing preventive knee braces, studied the patterns by which 47 of 100 injuries occurred to unbraced knees, and identified several extrinsic, sport-specific risk factors shared for both braced and unbraced knees. The attendance, brace wear choice, position, string, and session of each participant were recorded daily; medial collateral ligament sprains were reported whenever tissue damage was confirmed. Both the likelihood of wearing braces and risk of injury without them was highly dependent on session (games/ practices), position group (line, linebacker/tight end, skill), and string group (players/nonplayers). Subjects wearing braces often faced a high injury risk to their unbraced knees, a finding compatible with the opinion that braces were a necessary evil, best worn when concern over danger of injury outweighed desire for speed and agility. It is concluded that to avoid misinterpretations due to the confounding influence of brace wear selection bias, accurate investigation of daily brace wear patterns is required. Then, before considing the impact of preventive knee braces, a repartitioning of the data base is essential to assure that only similar groups will be compared.
\end{abstract}

†Address correspondence and reprint requests to: John Albright, MD, Sports Medicine Services, Department of Orthopaedic Surgery, University of lowa, lowa City, IA 52242.

No author has commercial affiliation with any product used in this article, nor has any author or related institution received financial benefit from any product. See "Acknowledgments" for funding information.
After more than a decade of investigating the effectiveness of off-the-shelf knee braces, controversy still exists as to whether they are the solution for preventing medial collateral ligament (MCL) sprains in football. ${ }^{1}$ The fact that the confusion has lasted this long is a reflection of the degree of difficulty inherent in doing the epidemiologic studies of sport-related injury patterns in general, and of team sports-where the interplay of all of the influential factors make the understanding of injury patterns a most demanding challenge-in particular. To further complicate the issue, football is the most complex of all team sports. One major pitfall in the process of establishing an overall injury profile has been the need to consider factors that may make one player more at risk for an injury than another. While these inherent factors do exist, it is the extrinsic (e.g., environmental or sport-related) factors that are the focus of this paper.

We believe that knowledge of the existence of these extrinsic factors is essential to the accurate interpretation of epidemiologic studies, and that failure to appreciate them can produce conflicting results. The purpose of this report was to support this contention by analyzing those factors that must be accounted for when studying knee brace effectiveness in football. To do so, we will describe the daily brace wear patterns, identify which players who do not wear braces are most likely to incur an MCL sprain of the knee, identify those extrinsic factors that influence this injury pattern, and assess the importance of the relationship between unbraced knee injury patterns and brace wear tendencies among study participants.

\section{BACKGROUND}

The Big Ten Conference is composed of 10 Division I NCAA universities, each of which plays a minimum of 10 football 
games per year. The members of the Big Ten Conference Sports Medicine Committee initiated a cooperative effort intended to study MCL sprains of the knee in a manner that addressed the pitfalls that had been observed to hinder previous research efforts. ${ }^{5}$ We first conducted a 1-year pilot study to refine the focus of our formal effort, strengthen weak spots in our recording system, and generate a critical level of enthusiasm among the investigators for undertaking the task. In this initial, descriptive phase of the study, all of the 1133 players listed on the football team rosters of the member institutions were included in the surveillance. Each player was identified on the roster as a person who either did or did not wear braces. The players were monitored for knee sprains during the 1984 fall season, with each medical team using the same 1-day time-loss criteria for a reportable injury as they had used during participation in the National Athletic Injury/Illness Reporting System (NAIRS). ${ }^{3}$ It was also noted on each injury report whether or not the player was wearing a brace at the moment of injury.

There were 186 time-loss knee injuries reported during the pilot study season. Included were 80 MCL sprains, 19 ACL sprains, 18 meniscal tears, and 69 other assorted maladies (e.g., contusions, abrasions, bursitis, other ligament sprains). The conglomerate frequency of all types of knee injury occurring to braced players was $18.1 \%$ (99 of 544 ), compared with $14.7 \%$ ( 87 of 589 ) of the players who were listed as wearing no braces. When looked at without qualification, MCL sprains also appeared to be less frequent among players without braces: $5.8 \%$ (34 of 589) compared with $8.5 \%$ ( 46 of 544 ) occurring in players who wore braces.

While these figures appeared to imply a greater risk of injury when braces were worn, this was at odds with the personal experience of most of the investigators as well as the fact that, as had been consistent for many years, nearly one half (544 of 1133) of the conference football team members chose to wear braces. Given the consistency of the observation that no player wore braces all of the time, a study subject "susceptibility bias" was thought to affect the patterns of which participants were choosing to wear braces during the pilot year. Many of those players interviewed admitted to applying or taking off their braces during the middle of game or practice sessions, depending on their concern for injury. Furthermore, there were great interinstitutional and intrasquad differences in when, and under what conditions, the prophylactic knee braces were being worn.

An informal review of conference-wide experiences led to two observations. First, there was a consistent pattern to the brace wear choices of the study group participants. Second, those players who were the most likely to choose to wear braces perceived themselves to be in situations of high risk for incurring a knee injury. On the one extreme, players in skill positions indicated that they did not feel they were very likely to incur a knee injury. They were most concerned that the braces would impair their speed and agility. On the other hand, those players in offensive and defensive line positions were very concerned about injury and were in the group that most frequently chose to wear braces. Some players who perceived themselves to be in the greatest danger often had even requested braces that were more sturdy than those available for prevention. Furthermore, on most teams, reports of converts to believers in wearing braces were particularly notable after a knee injury occurred to a position mate.

From review of this anecdotal experience, it was concluded that the players generally believe that braces are a necessary evil, best worn in times of great danger of injury. It was also hypothesized that the perceived injury risk associated with position, string, and session appeared to significantly influence the brace wear preferences of individual players. Armed with these pilot study findings, the Big Ten Sports Medicine Committee implemented a 3-year study. Intending to minimize unwanted variables and maximize uniformity of recording between institutions, we limited the focus of the study to the injury that the brace had originally been purported to prevent: MCL sprains. ${ }^{1}$

\section{MATERIALS AND METHODS}

The knee brace study protocol was superimposed on the recording activities of the ongoing, multisport Big Ten Conference Sports Injury Surveillance System. Institutional participation was voluntary and required that a daily log be maintained for all study group participants. To be eligible for the study roster, the athlete had to be free of a previous injury to either knee as determined by 1) no history of injury that caused a time loss of more than 10 days, or 2) no pathologic laxity noted on preparticipation screening examination for ligament integrity. These exclusion criteria were based on the observation during the pilot study that major knee sprains often appeared to have impaired the subsequent speed and agility of some athletes and may have even increased their susceptibility to another injury. Additionally, players with a history of serious sprains (e.g., requiring surgical reconstruction) appeared much more likely to wear a brace than those without such a history.

Confirmation that a reportable MCL sprain had occurred was the combined responsibility of the team athletic trainer and the team physician and was based on the determination of tissue damage, whether or not any time lost from action was associated with the injury. The clinical criteria uniformly employed for establishing the diagnosis included identification of a site of maximum tenderness over the anatomic region of the MCL and pain elicited in the same location with valgus stress applied to the knee flexed $20^{\circ}$ to $30^{\circ}$.

From the experiences of the pilot study, it was clear that a method for counting the actual days the braces were worn by each study group participant had to be implemented to better evaluate the efficacy of the prophylactic braces. Therefore, the number of high-injury risk sessions they participated in and whether or not they wore a brace were recorded daily for both knees of every player in the study. For the purpose of this report, a preventive brace was defined as any device designed specifically to provide protection from a lateral blow to the knee that may result in an MCL sprain. As opposed to the customized, "functional" 
braces prescribed after rupture of an ACL, the preventive brace is usually an off-the-shelf device with single or double uprights. While there were a wide variety of preventive knee braces worn by the subjects of this study, no further details will be pursued because that is not pertinent to this paper. Each player's record also included his position, string, and a categorization of the type of session scheduled.

\section{DEFINITIONS}

Because many of the terms involved in the influential factors that were studied are esoteric to either the sport or the investigation, a brief definition of terms is warranted.

\section{Session}

Within the different sessions associated with college football (e.g., games and practices) there are a variety of patterns of injury risk. Intercollegiate games, where only a limited number of team members participate while others stand and watch, are the most intense and the most consistent sessions. Although never close to matching the level found in games throughout this study, the intensity of practice session exposures varied considerably between institutions, within each institution (e.g., according to coaching philosophy, time of year, won-loss record, cumulative number of injured for the season), as well as within each team, among different string and position groups. However, pilot study and surveillance system information indicated that it was possible to divide these planned sessions into categories based on the relative likelihood that contact injuries would occur.

The term "contact practices" included all practice sessions of interest for this study. The degree of intensity of the contact practice session varies according to the following three categories: Formal "scrimmages" are game-like practice sessions that, during the fall season, are usually held for team members who are not likely to get in the Saturday game. These are very intense, high-injury risk sessions because the participants are trying to impress their coaches to be allowed to play in the games. "Regular practice" describes the team's routine weekday contact practice, which may include periods of full contact scrimmage. However, the contact is usually controlled, perhaps even with certain rules such as a restriction from contacting the quarterback and avoiding "piling on." In either instance, scrimmages were included in this study as contact practice sessions. "Light/noncontact practices" are designed for conditioning or rehearsal of execution, and are associated with little contact. These were not included in the calculations in this study because preventive brace wear, as well as the occurrence of knee injury, is minimal during these times. Inclusion in the study would have added more practice sessions, which would have biased the results in favor of not wearing a brace. The terms "athlete" and "knee exposures" as used in this study express the number of high-risk opportunities for incurring a football-related MCL knee sprain that study subjects, or their two knees, experienced by their participation in either game or contact practice sessions.

\section{String}

This term is a designation for the rank ordering of the several players assigned to each position, according to the coach's assessment of skill level, listed from best to worst on a depth chart. In the pilot study, we established a case for separating the athletes by string groups because there appeared to be variations in game and contact practice exposure patterns, unbraced knee injury rates, and brace wear choices, according to assigned rank order status on the depth chart. Football team members are usually referred to as being on the first, second, third, and so on string; for the purposes of this study, they were divided into "players" and "nonplayers" according to the likelihood that they would participate in the critical moments of a game and that the practice sessions would be designed to prepare them to meet the upcoming opponent. The players group consisted of "starters" and "substitutes". Starters are the best players in their positions and are expected to start in each game and play most or even all of it. Their contact during the practice sessions may be limited more than others behind them. Substitutes are players on the second and third teams who are prepared to play in each game. While their actual game exposures may range from very limited to as much as $50 \%$ of the total number of plays, their practices are geared toward improving technique and skills in preparation for the next game in a fashion that is similar to that of the starters. Nonplayers are team members who are not expected to play in games. Their usual role is to provide the players a chance to rehearse against a surrogate of the next scheduled opponent. Nonplayers usually see more contact than their higher ranked peers during regular practices as they must repeatedly demonstrate the anticipated tactics of their upcoming opponents until each of the players appears adequately prepared to succeed in winning the game. The highest injury risk contact practice sessions that nonplayers participate in are the three to five previously mentioned formal scrimmages. While not adequately accounted for by the grouping employed for this report, players assigned to the lower end of the substitute group (e.g., third and fourth strings) were often recorded as having also participated in the formal intrasquad scrimmages.

\section{Position}

The traditional positions associated with football were used to identify each participant in this study as they are usually found in a game day program. This classification contains variations in the assigned tasks. The "line positions" group consisted of individuals who usually start each play on the line of scrimmage, bent down in a three-point stance. The "skill positions" group contains those position assignments that require the greatest amount of speed and athletic ability. The "linebacker/tight end positions" group is connected by the fact that on running plays, players in these positions must compete with linemen, and on passing plays they must keep up with the skill positions group subjects. 


\section{DATA ANALYSIS}

To demonstrate the magnitude of the impact that extrinsic, sport-related factors will have on studies of knee brace effectiveness in college football and to familiarize the reader with the process of analysis, the approach taken in this study has been to limit the data analysis to a practical sequence of graphic and other elementary interpretive techniques for obvious relationships that can be graphically validated. The insight gained from this effort is also essential for understanding the multivariate analysis techniques that should be required for validation of future descriptive studies of preventive knee brace effectiveness in intercollegiate football. Because of the daily attendance and brace wear records available for each individual in this study, a second perspective is also made possible. Such detailed records allow serial looks at the record of each study subject's brace wear pattern, position, string, and session as it relates to the number of mean exposures before either incurring an MCL injury or leaving the team for some other reason.

While survival analysis techniques have become popular in total joint replacement procedures and industrial job safety records, this approach has been mentioned ${ }^{8}$ but not previously applied in the field of sports medicine epidemiology, and the ramifications and nuances of the analytical procedure are presently unexplored. The introduction to this type of analysis and sharing of the information available is intended to provide for the further development and standardization of the procedure.

\section{RESULTS}

\section{Injury surveillance system background statistics}

During the 3 years of this special study on MCL sprains and preventive knee braces, an ongoing, multiple sport injury surveillance system also continued to concurrently gather information on all time-loss injuries and illnesses for all players on the football team rosters of all 10 institutions. Reports from that system indicate that there were study period totals of 3720 player-seasons and 292,430 athlete exposures. The average conference team consisted of 98 members participating in 93 regular practices and 3.7 formal scrimmages per year. In addition, each team consistently played an average of 53 team members for 11.5 games, creating 106 knee exposures per game. Twenty-five percent of the football players in the Big Ten Conference were classified as starters, $27 \%$ as substitutes, and $48 \%$ were listed as nonplayers.

Over the 3-year study period, there were no detectable variations in the number of time-loss injuries reported through the Big Ten Conference Injury Reporting System, with 2 schools reporting more, 2 less, and 6 maintaining the same number of injuries. There were 2608 reported injuries to all body parts; these were consistently distributed over each of the 3 years, with a range of 9 to 12 injuries occurring per 1000 athlete exposures. Among the more common injuries were 475 knee injuries, 392 ankle injuries, 120 foot injuries, 198 neck injuries, and 151 head injuries. Of the total 475 knee injuries, 205 (43.1\%) involved the MCL. The rank order list of MCL injuries occurring by position includes offensive line (47), and the defensive line (30) at the top and the kickers (2) at the bottom. Of the $205 \mathrm{MCL}$ injuries, 100 occurred in study group participants and 105 occurred in the nonstudy team members.

\section{The study group}

Nine of the 10 conference member institutions supplied the data for 23 of a possible $30(77 \%)$ team-seasons. Five institutions met all reporting criteria for all 3 years. Four institutions had valid data accepted for only 2 of the 3 years because they failed to provide complete and accurate daily team rosters for an entire fall season. During the 3 fall seasons studied, there were 987 different varsity football players who qualified as study group participants and had completed daily records. The number of qualifying participants turned in by schools ranged widely (Fig.1). The fewest number of study group participants reported from 1 school in any 1 year was 40; the maximum was 83 .

Except for their lack of a previous knee injury, the study population was representative of the entire population of conference football team members. This was particularly true for the distribution of players by string and position in that, except for offensive backs, the subgroup sizes did not vary proportionately from those of the whole team by more than $1 \%$ to $3 \%$. The offensive backs were underrepresented, comprising only $7 \%$ of the study group, whereas this group made up $14 \%$ of the entire team.

\section{Athlete exposures}

Cross-sectional analysis. The total number of contact practice sessions and games in which the attendance of the study group subjects (athlete exposures) exposed them to a risk of a knee injury (knee exposures) ranged widely among schools. However, it was fairly consistent for the entire conference in each of the 3 years that were studied. With many subjects continuing in the study more than 1 year, this group of 987 athletes accounted for a total of 1431 player-seasons. This group accumulated 77,861 athlete exposures or 155,722 knee exposures by participating in

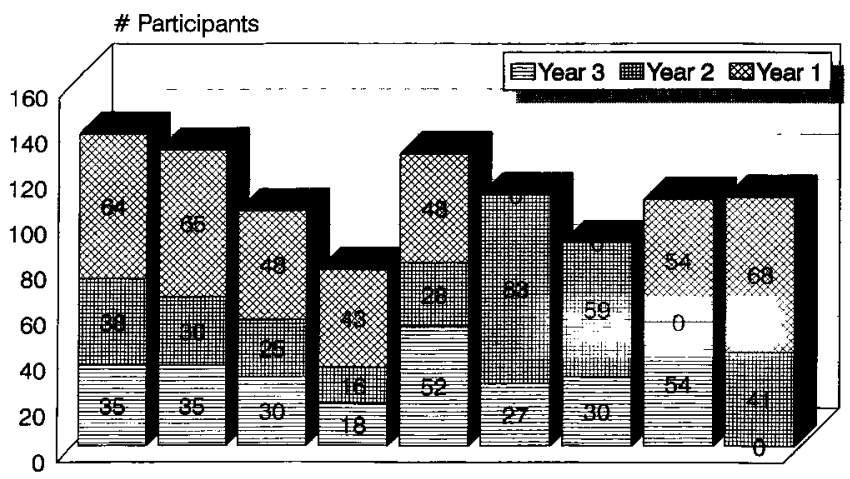

Participating Institutions

Figure 1. Study group size, by institution. 
high-injury risk sessions involving varying degrees of physical contact that were scheduled for their respective football teams. The number of game exposures was greater for those teams invited to postseason bowls, but was otherwise fairly consistent because of the NCAA limits on the size of the squad that could dress for games at home and away. On the other hand, the number of contact practice exposures depended on whether or not the team had extra postseason practices before a bowl game. This figure also reflected the number of participants enrolled in the study at each institution and was most heavily influenced by the size of the nonplayer group.

Serial analysis. Many players remained participants in the study for more than 1 year before either incurring an MCL sprain or terminating participation in football for any other reason. More specifically, $64.3 \%$ of the players remained in the study for only 1 year, $26.3 \%$ participated for 2 years, and $9.3 \%$ for 3 years. The size of the study group was relatively even over the 3 -year period. The number of player-seasons were 388 in the 1st year, 318 in the 2nd year, and 218 more in the 3rd year. Of the original 388 players that started in the 1st year of the study, $92(23.7 \%)$ were still around to have begun all 3 years with their names listed on the daily attendance and brace wear roster, and $76(19.6 \%)$ were the survivors (given that they remained academically eligible and did not drop out for any other reason), who remained totally unscathed by knee injury for the entire 3 years of the study.

\section{Brace wear patterns}

For the entire study group, 78,911 (50.7\%) of the knee exposures were with prophylactic knee braces and 76,811 $(49.3 \%)$ were without any braces. The pattern of where, when, and how often an individual participant chose to wear braces most closely paralleled his teammate playing the same position on any conference team. Furthermore, for the purposes of data analysis, there was sufficient similarity in the brace wear habits of different positions that they could be divided into three position groups (Fig. 2). Given the mean values for each of the position groups, the two other extrinsic (sport-related) factors that obviously related to the frequency of brace wear across the entire

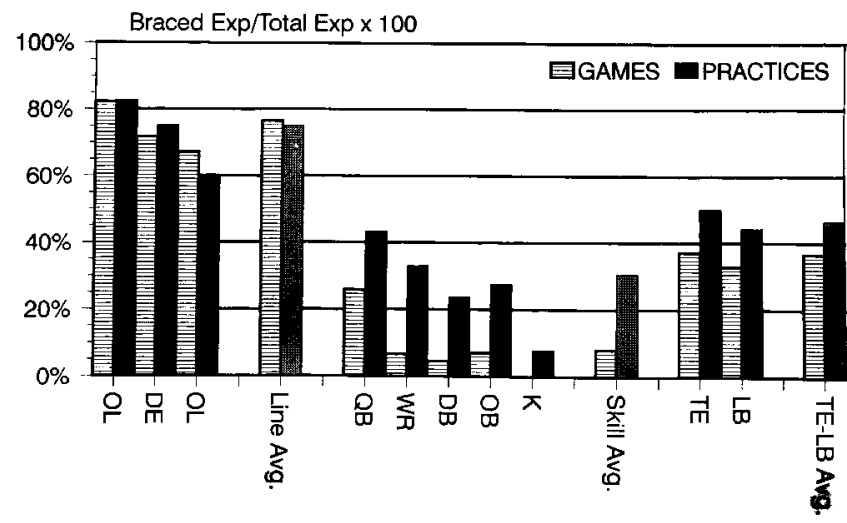

Figure 2. Brace wear frequency by similarity of positions. study subject population included the type of session that was scheduled and the string that each study subject was assigned.

Brace wear by session. None of the study subjects wore braces in any of the light or noncontact practice sessions. While these sessions were eliminated from any of the calculations reported here, they amounted to nearly $15 \%$ of all practice-related athlete exposures. Even when looking at sessions where there is an increased injury risk, no player in the entire study wore knee braces for every session. However, $373(37.8 \%)$ study subjects went to one extreme or another in their brace wear tendencies. There was a group of 148 study subjects who chose to wear their braces during more than $90 \%$ of the high-injury risk sessions. On the other extreme, there were 225 study subjects who wore their braces less than $10 \%$ of the time.

Overall, while $52.6 \%$ of the knee exposures were in braces during contact practice sessions, only $36.7 \%$ of the knee exposures were in braces during games. It is also of note that, because of a given inequality in the number of scheduled practices over the number of games, there were many more knee exposures accumulated during practices than games; $91 \%$ of all of the braced exposures occurred in practice, as did $84.2 \%$ of all of the unbraced exposures.

Brace wear by position. Of the 148 high brace wear subjects (more than 90\% of knee exposures), 99 (66.9\%) were from positions in the offensive and defensive lines, 28 (18.9\%) from the linebackers and tight ends, and $19(12.8 \%)$ from the backs, receivers, and kickers. The makeup of the 225 study subjects in the low brace wear category was a mirror image of the high brace wear group, namely 162 (72\%) were backs, receivers, or kickers; 34 (15.1\%) were either linebackers or tight ends; and 29 (12.9\%) were from the offensive and defensive line positions. From this polarized view of brace wear tendencies alone, it was obvious that linebackers and tight ends wear braces selectively, as these 2 positions had the fewest subjects who went to one extreme $(90 \%)$ or the other $(\mathbf{1 0 \%})$

Verifying that it was not just a few extremists who tended to be assigned to certain positions on the team, the partitioning of study subjects into these position groups by general tendencies followed a pattern that was identical to the polarized data partitioning. Whether or not the scheduled high-injury risk session was a contact practice versus a game appeared to affect the brace wear tendencies of each position group differently. Study subjects in the line positions group tended to wear braces nearly $75 \%$ of the time, in both games and practices. The study participants in the skill positions group all shared a characteristic of wearing braces the least often and with a greater regularity in practices $(26.1 \%)$ than in games $(10.2 \%)$. Within this group, the single position where participants were most likely to wear a brace in game or practice was at quarterback. The two least likely individual positions within this group to find braces being worn were wide receiver and kicker. The members of the third position group shared a characteristic of always remaining relatively equally divided about the value of wearing braces. Fifty percent of the linebacker/ tight end group wore braces in practice, but only $40 \%$ wore them in games. 
Brace wear by string. Because of the similarities between the brace wear patterns (Fig. 3), separating the three string groups into players (starters and substitutes) and nonplayers appeared to best separate the different types of roles at games and contact practices within all three of the position groups. At each of these position groups, there are generally different patterns of knee exposures between the players and nonplayers with and without braces as well as during both games and practices. More specifically, in practices, while the effects of the position that the study subjects were assigned to were obviously more pronounced, knee braces were consistently more common on nonplayers at all three position groups. On the other hand, in games, players in the line positions wore braces slightly more often than in practice and much less often in the other two position groups. There was a marked tendency for players to shed their braces for games. This was most dramatic in the skill positions group ( $30.4 \%$ versus $8.0 \%$ ), but was also present in the linebacker/tight end group $(46.4 \%$ versus $36.6 \%$ ).

\section{Injuries}

There were a total of 182 knee injuries among the members of the study group, with 100 involving the MCL. This left 887 participants in the study who did not incur an MCL sprain. In the group of 100 subjects with MCL sprains, there were also 11 players who further incurred an MCL sprain to the other knee after they were dropped from the virgin knee study after their first injury. Their data for the second knee injury have not been included for analysis.

Injuries by session. It was the type of session that affected the MCL injury rate of the unbraced knee to a greater extent than any other factor (Fig. 4). The fact that there was a nearly equal total number of injuries that occurred in practice and games (52 versus 48 ) is accounted for in the injury rate calculation because there were 9 times as many practice sessions scheduled.

Injuries by position. Second only to the type of session in terms of strength of influence, the injury rates for the unbraced knee exposures were greatly influenced by the position being played. In contact practices, the rate of injury for players without knee braces was equally high for the line and linebacker/tight end position groups and lowest for

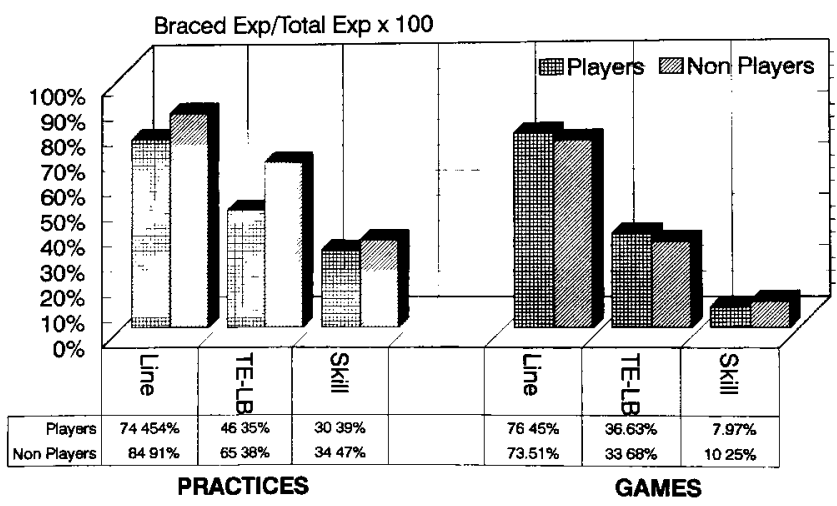

Figure 3. Brace preference, by string.

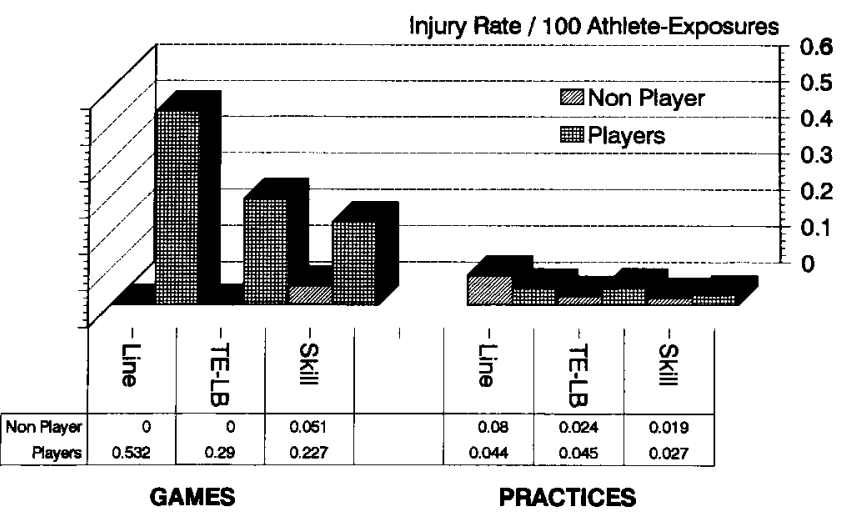

Figure 4. Unbraced injury rate.

the skill positions group (Table 1). During games, an increased risk of injury was experienced by all position groups, but the greatest change was noted in the line positions group.

Injury by string. Another obvious factor influencing the risk of MCL sprain faced by a study subject is related to where they were placed on the depth chart by the coaching staff. Since the results were quite different in direction and magnitude, they are presented separately.

Injuries in players. In practices, the rate of injury for the players not wearing braces in the line and linebacker/tight end positions group was roughly 1.7 times greater than for the skill positions players. During games, a dramatic pattern emerges for each of the respective position groups where an increased risk of injury ranges from a low of 6.4 (linebacker/tight end positions) to 8.4 (skill positions) to a maximum of 12.1 (line positions) times the injury rates experienced during practices. Given that everyone participating in a game is at his greatest risk of injury, at 0.5 injuries for every 100 knee exposures, starters and substitutes in the line positions group are nearly twice as vulnerable to MCL sprains during the course of a game as those players in the other two position groups (Table 1).

The serial assessment of how many high-risk sessions the individual study subjects survived without braces is of interest. For the 521 subjects in the players string group, $260(49.9 \%)$ of the individuals remained in the study only 1 year. Sixty-nine $(26.5 \%)$ of these were eliminated from the study because of an injury to the knee. The remaining $191(73.5 \%)$ players dropped out of the study group for other reasons (e.g., graduation, quit team, end of study period, career-ending injury of another body part). As a group, those in the players' string group who were eventually injured without knee braces accumulated a mean of 138.9 knee exposures before their injuries. Those in the line positions group survived the greatest number of high-risk exposures (156.4 unbraced knee exposures in contact practices and games) before injury, while the linebacker/tight end group lasted the shortest time at 123.4 athlete exposures. On the other hand, the skill position players participated in a mean of 138.8 athlete exposures before injury.

Injuries in nonplayers. Among the three position groups for the nonplayers, the same general rank order of injury 
TABLE 1

Position group totals

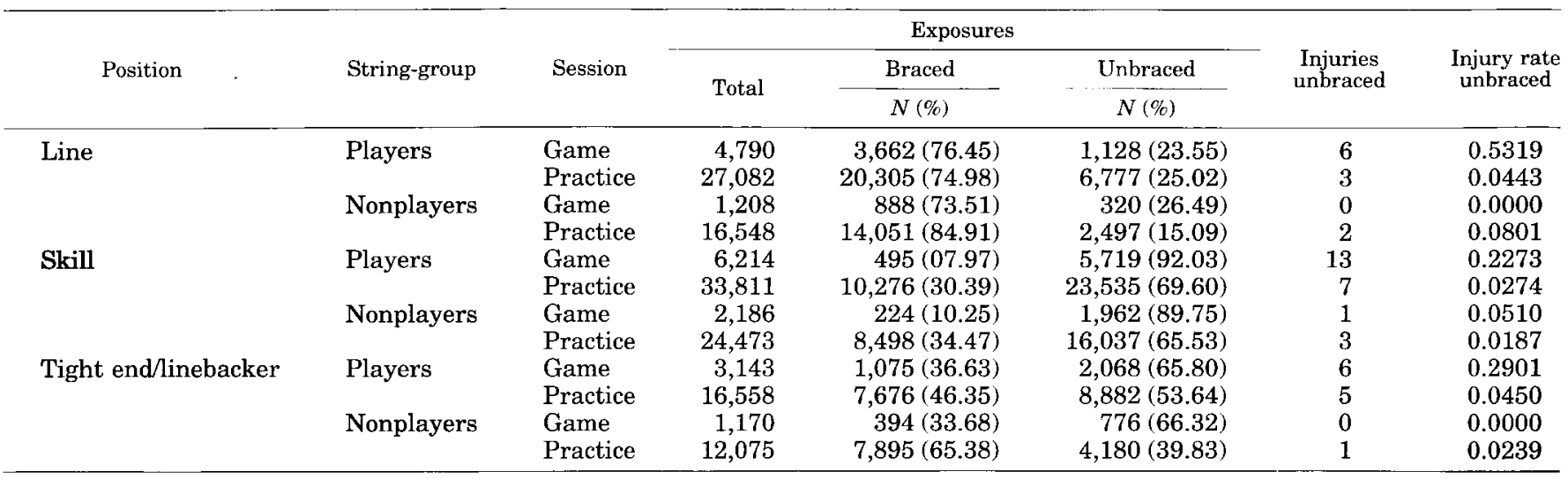

rates existed as with players, with the highest number in the line positions, followed by the linebacker/tight end positions and then the skill positions. In contact practices, it is the nonplayers in the line positions group that have the greatest injury rate of all of the unbraced team members. However, reflecting the fact that by definition nonplayers do not get into the critical parts of games, their injury pattern was practically nonexistent. Only two injuries (one of them to a kicker) occurred under these conditions with relative low exposure to game conditions

Serial assessments of the data for the individuals in the nonplayer group reveal a pattern that is different than that for the players (Fig. 5). While the high brace wear habits of this group meant that very few fit into this category, study subjects in the nonplayer division of the line positions group accumulated a mean of only 11 athlete exposures before injury when the brace was not worn. For linebacker/tight ends, the unbraced group had 26 athlete exposures before injury. The skill position nonplayers enjoyed a relatively long number of athlete exposures (85.5) before injury when the brace was not worn.

\section{Association of brace wear choice and unbraced injury rate}

By graphically comparing the values for players in practices, a strong relationship can be identified between brace

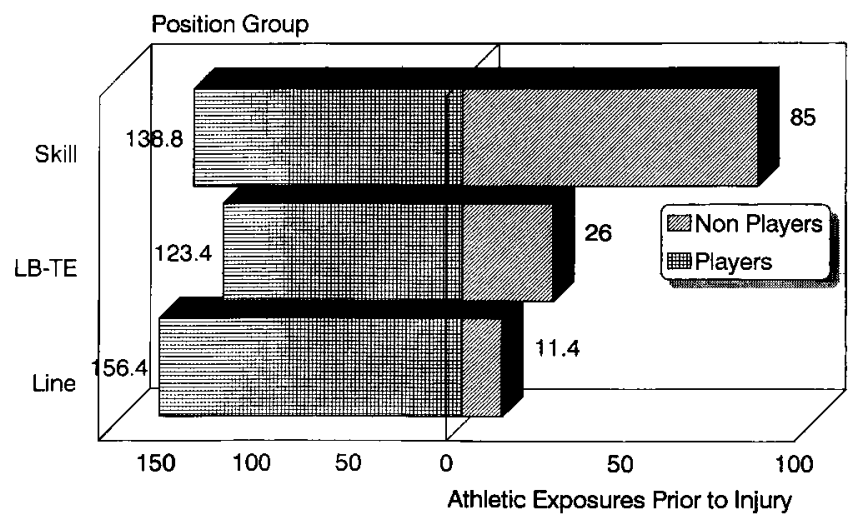

Figure 5. Time to injury, by position and string. wear preferences and risk of incurring an MCL sprain when braces are not worn (Fig. 6). While not exhibiting as strong an influence as session and position, to be aware of which string group a subject was assigned to proved to be critical. Even when comparisons were limited to study subjects in the same type of session and assigned to the same position groups, quite different patterns still appeared that were related to their rank order on the coaches' depth charts. For instance, in practice sessions, for each of the three position groups, the frequency of brace wear for nonplayers was consistently higher than for players, but the unbraced knee injury rate was only greater among those in the line positions group. This nonplayer division of the line positions group was particularly noteworthy because not only was their unbraced knee MCL sprain rate (84\%) highest of all groups during practices, but they also displayed the highest brace wear rates of all groups in both practice and games. On the other hand, in games, nonplayers in the line positions did not participate, were rarely injured, and wore braces less often than their position mates who as players were actually on the field competing against the players from the rival institution. Among the players (starters and substitutes), the highest popularity of the braces as well as the greatest injury risk for the unbraced knee was found in the line positions group (centers, guards, and tackles), followed by those in the linebacker/tight end

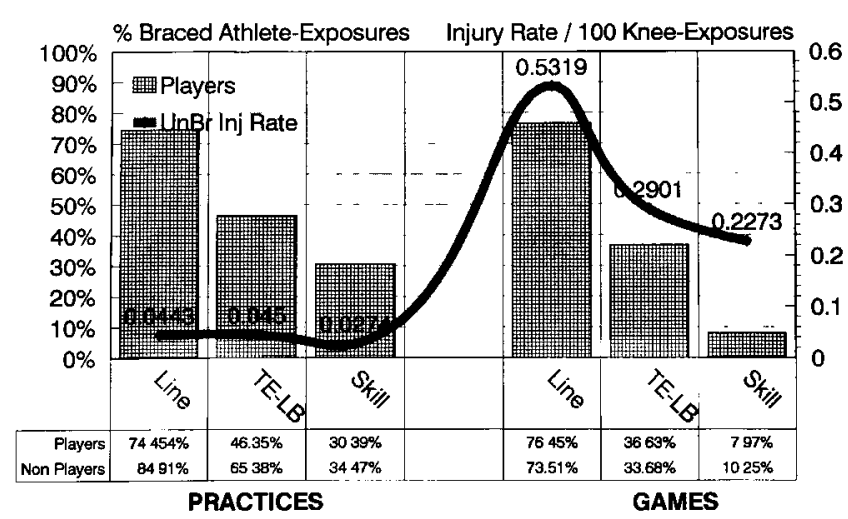

Figure 6. Brace preference versus unbraced injury rate. 
positions group, and least often by the skill positions group (receivers, backs, quarterbacks, kickers).

\section{DISCUSSION}

Except for their history of knee sprains, the athletes chosen for study were indeed representative of the entire population of conference football players. The fact that about one half of all conference football players qualified for the virgin knee study group is a confirmation that MCL sprains are all too common in this sport, making the value of putting forth the effort to reduce these types of injuries obvious. Furthermore, the insight provided by this communication provides compelling support to the suggestion that standards be set regarding future studies of this type. ${ }^{5}$

It is of note that while most $(78 \%)$ MCL sprains were classified as mild severity, there was also greater interinstitutional variation in the number of injuries reported at the lower levels of severity. There were also frequent incidences at each institution, in both the pilot and the formal studies, in which a "near miss" or a "save" was noted on only an anecdotal basis. This generally amounted to incidences where an athlete reported that there had been a blow to his knee of sufficient force to cause an MCL sprain were it not for some palliating circumstance (e.g., the preventive brace, wet grass, flexed knee position at impact). These are of note only from the standpoint that if the braces are actually maximally effective at turning a would-be mild injury into no injury at all, then the injury-reporting threshold must be lowered beyond the simple clinical establishment that MCL tissue damage had occurred. Documentation of the number of critical incidents where an MCL sprain might have occurred would require such techniques as multiple angle cameras, strain gauge, or accelerometer telemetry. While any of these methods may prove impractical, they would provide the ideal research tool for quantifying the exposure factor.

Analysis of brace wear effectiveness in any setting is best done in a well-controlled, prospective study with random selection of who does and does not wear braces. This type of scientific approach has only been performed by one research team to date. ${ }^{12}$ Because of the impracticality of enforcing the experimental format in organized athletic competition, most studies have and will continue to be confined to descriptive-type studies where there is no control over who wears braces. Given this latter situation, efforts to minimize the chances of misinterpretation require a routine that first provides daily, or even more precise, detailed accounts of brace wear and opportunities for injury (exposures) for the more influential factors of session, string, and position. Then, before any statistical assessment of the preventive impact of knee braces is begun, the original data base must be reorganized in a way that allows dissociated analysis of each factor under consideration in a step-bystep fashion.

Both simple and sophisticated multivariate analysis formulas are of little or no value until the data base has been properly partitioned. Because of the limitations of numbers, this process will be feasible only after individual players are grouped together by position and string, where they share similar job descriptions, body types, and brace wear patterns. For example, in studying protective equipment in Division I college football, players should be analyzed separately from nonplayers. Nonplayers should be analyzed only during contact practice sessions. Players can be studied during contact practices and games, but the distinction between these sessions must be consistent in the analysis. Furthermore, before analysis, individual positions should be divided into three general groups (e.g., line, skill, and linebacker/tight end positions).

The light/noncontact practice sessions were not included in the knee-exposure calculations in this study. There were $15 \%$ of practices where no braces were worn because no contact, and thus no risk of knee injury, was expected. The knee exposures of the nonplayers were also not included at all in the denominator calculations for games and only as a separate group during practices. This was justified by the consensus of the investigators that, given their quite different patterns of injury and brace wear habits, to do so would otherwise be misleading when the effectiveness of preventive bracing was the issue. They were excluded by the string group definition that nonplayers were those study subjects who did not get to play in the critical parts of games. This meant that the only time they would see action in a game was when there was such a discrepancy in the score that the home team was able to clear the bench. Because of NCAA rules about the size of traveling squads, nonplayers are not taken to games played at the opponent's institution. With nonplayers standing on the sidelines at home games rather than actively participating, the injury rate for this group was essentially zero and their tendency to wear braces was lower in the line and linebacker/tight end positions, while not quite as low as $8 \%$ of the starters and substitutes in the skill positions.

The two string groups were considered separately in practices. Of special interest during practices is the nonplayers' division of the line positions group where the brace wear popularity ( $85 \%$ knee exposures) was the greatest of all for any group regardless of session, while their unbraced MCL sprain rate was nowhere near that of their position mates who were the starters and substitutes during games. The informal pilot study interviews led to the opinion that this group was often the most concerned about their vulnerability to injury. This concern may have been related to their regular practice role of simulating the next opponent and when they were given a chance to show their talents to the coaching staff in the few formal intrasquad scrimmages held each fall for their benefit. Given the high brace wear frequency of this group, the differences between the expected and actual injury rates may also indicate that a protective effect of the knee braces was being realized. Regardless of speculations in either direction, it was clear that without proper partitioning of the knee exposures of the entire study group for the light/noncontact practices and of the nonplayer string group during games and practices, an automatic bias occurs. While differences in approach make the issue impossible to resolve, it is important to point out that the bias noted in this report is of such magnitude that correcting for it before running the standard statistical analysis packages could possibly even 
change the results of those studies where it was concluded that wearing the brace may cause an increased risk of knee injury. ${ }^{6,7,11,14}$

Consistent with other reports ${ }^{5,6,7,10-12,14}$ the brace was found to be predominantly worn by offensive linemen, followed by defensive linemen, linebackers, tight ends, and then all categories of backs. However, the meticulously gathered denominator information about daily exposures to injury and brace wear is necessary to provide a precise knowledge of when and how often players actually chose to wear braces during any one season. From the pilot study observations, it can be hypothesized that the deciding factor that dictates whether or not a brace is to be worn depends on which concern (e.g., perceived risk of injury and urgency for uninhibited performing speed and maneuverability) is momentarily more dominant in the athlete's or his coach's mind. Even the lighter, single upright preventive braces are universally considered by the players to be necessary evils. Therefore, in those situations where the competitive edge includes maximizing speed and agility, the urge to not wear a preventive brace is the greatest. Pilot study questioning indicated that, in general, it was believed that players' choices related to the perception that the braces are associated with a notable performance reduction including speed, strength, and agility. This perception has some support in the recent literature. ${ }^{4,9}$ Studies by Styf et al. ${ }^{13}$ revealed that functional knee braces increase intramuscular pressures at rest and muscle relaxation pressure during exercise to levels that decrease muscle blood flow to the degree that they may lead to early development of muscle fatigue. Apparently, the calf pressure is directly related to the tightness of the elastic straps.

A belief in the effectiveness of the preventive knee braces is critical to their being used at all. That this belief remains strong is evidenced by the surveillance system observation that the frequency of use had not diminished in the conference before, during, or subsequent to the years of the study. Further evidence surfaces by comparing the circumstances for each of the 3 position groups for practices and games. For those players in the line positions group, the rate of injury to the unbraced knee is highest on the team, greater in games than practice, and the most consistent between them. It is also this same group that exhibited the highest and most consistent brace wear rate on the team. The vigor for brace wear in this group was repeatedly documented to increase after a fellow lineman had incurred an MCL sprain. It was for an even more compelling reason that 11 of the $110 \mathrm{MCL}$ sprains actually occurring to the study population during the study period were eliminated from consideration. These participants were injured twice but the second injury was not included in this study. The daily attendance records show that they were not wearing a brace until they incurred the first MCL sprain. The decision to drop the second injury from the study was made on the basis of the pilot study findings that the first injury had been noted on occasion to visibly impair the agility of the athlete at least temporarily, therefore adding another factor that made that player potentially more susceptible to injury. It was also a pilot study observation that perhaps an even greater influence (as opposed to injury to a position mate) on the conversion of an athlete from the ranks of the unbraced to one of the most faithful brace wearers was experiencing an MCL sprain in his own knee. This combination of increased injury risk and brace wear exposure could obviously bias the results. To what degree, or even whether or not, either altered injury risk or brace wear conversion actually occurred to these 11 players during the study is not certain because daily roster information was not continued once an MCL sprain was reported.

Among players in the linebacker/tight end positions, the unbraced knee MCL injury rate is also high. While these players are at a greater risk of injury during the games, they show a weaker but still-present preference to remove their braces for games. It appears that, particularly during games in those two positions, the desire for maximal speed and maneuverability is overwhelming despite the potential for injury. Their choice is made because of their concern with maintaining maximum speed and agility on those plays where they must match faster and more agile players from skill positions. ${ }^{11,12}$ We believe that the skill position players are most likely to sacrifice safety for speed and agility. On the other hand, it will be up to future investigations to determine why they do not all refuse to wear braces. Who is it in this group who has chosen to wear a brace rather than have maximum speed and agility? Did his brace wear choice involve a known danger? For instance, as it is the quarterbacks that are most likely to wear a brace, is it a quarterback on a passing-oriented team with a losing record about to face the team with the best defensive statistics in the nation who feels the need to wear his braces?

\section{CONCLUSIONS}

Like most injuries in sports, MCL sprains are the result of a variety of factors that exist at the time of the injury that can be recorded. In an effort to minimize the chances of misinterpretation, the original data base must be reorganized in a way that allows separate analysis of each of these factors under consideration in a step-by-step fashion. Both simple and sophisticated multivariate analysis formulas are of little or no value until the data base has been properly partitioned. Because of the limitations of numbers, this process will be feasible only after individual players are grouped together by position and string where they share similar job descriptions, brace wear patterns, and risk of injury to the unprotected knees. Before even considering the impact of preventive knee braces, the confounding effects from each of the influential factors must be identified. Once this has been accomplished, the original question then changes to, Given that all else is equal, is the brace effective? It is suggested that future investigators of knee brace effectiveness should keep track of the number of consecutive high-risk exposures each study participant completes free of injury, as well as calculate the injury/ exposure index for both injured and uninjured study subjects to evaluate the value and practicality of this analytical technique.

Descriptive studies on the effectiveness of braces in the prevention of MCL sprains of the knee in football should reconcile the suggestion arising from this study that when athletes or their coaches are allowed freedom of choice, an 
inherent selection bias is generated from their beliefs that the braces are protective but performance-limiting devices. Even when confining the sessions to high-injury risk situations (e.g., contact practices and games), football does not present each player with the same risk of knee injury. Therefore, accurate investigation of daily brace wear patterns is required to first assess the influences of these factors and then repartition the data base to assure that similar groups are being compared. Among the more influential sport-related factors as to whether an unbraced Division I college football player is at risk for incurring an MCL sprain are session, position, and string.

The intensity of the type of session scheduled is the most important determinant of the MCL sprain rate in football. During games, injuries occur at a rate that is about 6 to 12 times greater than the rate associated with practices, and the patterns of injury are different than in practices. In a noncontact practice there is virtually no risk of an MCL sprain, and knee braces are not believed to be necessary. Therefore, including these noncontact practice sessions in the data base as practice knee exposures should be avoided. The results would otherwise be misleading by being slanted in favor of the safety of not wearing a brace. Individual positions should be divided into 3 general position groups (e.g., line, skill, and linebacker/tight end positions) according to similarity of brace wear preference patterns in practices and games.

Finally, because they demonstrate similar brace wear preferences in contact practices and games, players (starters and substitutes) should be analyzed separately from nonplayers. Nonplayers should be analyzed only during contact practice sessions. Players can be studied during contact practices and games, but the distinction between these sessions must be consistent in the analysis.

\section{ACKNOWLEDGMENTS}

This study was sanctioned and funded by the Big Ten Conference. The authors thank the following persons for their contributions to this study: athletic trainers-John Oschenwald, Danny Foster, Larry Leverenz, Sally Nogle, Paul Schmidt, Pat Beckmann, William Davis, Sandra Breitlesbach, and Matthew Carter; physicians-John Miller, Robert Gurtler, Herb Ross, Dave Hough, Doug McKeag, Gerald O'Conner, Edward Wojtys, Howard Sweeney, Gregory Palutsis, Robert Murphy, Loyal Combs, Steve Badyiak, Gregg Landry, William Clancy, and Pat Smith.

\section{REFERENCES}

1. Anderson G, Zeman SC, Rosenfeld RT: The Anderson Knee Stabler. Physician Sportsmed 7(6): 125-127, 1979

2. Brown TD, Van Hoeck JH, Brand RA: Laboratory evaluation of prophylactic knee braces performance under dynamic valgus loading using surrogate leg model. Clin Sports Med 9: 751-762

3. Clark KS, Alles WR, Powell JW: An epidemiological examination of the association of selected products with related injuries in football, 19751977. Contract CPSC-C-77-0039, Washington, DC, US Consumer Product Safety Commission, 1979

4. Fujiwara LM, Perrin DH, Buxton BP: Effect of three lateral knee braces on speed and agility in experienced and non experienced wearers. Athletic Training 25: 160-161, 1990

5. Garrick JG, Requa RK: Prophylactic knee bracing. Am J Sports Med 15: 471-476, 1987

6. Grace TG, Skipper BJ, Newberry JC, et al: Prophylactic knee braces and injury to the lower extremity. J Bone Joint Surg 70A: 422-427, 1988

7. Hewson GF, Mendini RA, Wang JB: Prophylactic knee bracing in college football. Am J Sports Med 14: 262-266, 1986

8. Loosli AR, Requa RK, Garrick JG, et al: Injuries to pitchers in women's fast-pitch softball. Am J Sports Med 20: 35-37, 1992

9. Lundin $\mathrm{O}$, Styf $\mathrm{J}$ : Intramuscular pressures varies with the tensile force used at knee brace application. Trans Orthop Res Soc 18(2): 310, 1993

10. Randall F, Miller H, Shuur D: The use of prophylactic knee orthoses at lowa State University. Orthot Prosthet 37: 54-57, 1983

11. Rovere GD, Haupt HA, Yates CS: Prophylactic knee bracing in college football. Am J Sports Med 15: 111-116, 1987

12. Sitler M, Ryan J, Wheeler $\mathrm{JH}$, et al: The efficacy of a prophylactic knee brace to reduce knee injuries in football: $A$ prospective randomized study at West Point. Am J Sports Med 18: 310-315, 1990

13. Styf JR, Nakhostine M, Gershuni DH: Functional knee braces increase intramuscular pressures in the anterior compartment of the leg. Am J Sports Med 20: 46-49, 1992

14. Teitz CC, Hermanson BK, Dronmal RA, et al: Evaluation of the use of braces to prevent injury to the knee in collegiate football players. $J$ Bone Joint Surg 69A: 2-9, 1987 